ever possible. It is one of the most useful methods in treating those who have defective cerebral inhibition. Systematized labor properly directed will accomplish a double purpose. It will first of all encourage those patients who have sufficiently recovered to realize that the institution is managed from a humanitarian point of view; namely, to assist them in their present condition and after their return to their homes. In the next place, the institution from such an arrangement may become self-supporting. Social service is a useful adjuvant to such a plan. It will facilitate the return of the subject to a normal life by procuring him work, by properly advising him and by developing in him the spirit of hopefulness and initiation.

At the beginning of this article it was shown what preventive legislation may do. Reduction of the number of saloons may help to a certain extent, but such a procedure is considerably less valuable than reducing the production of alcoholic beverages, as the existence and increase of saloons are the result of a demand for alcoholic beverages. As legislation in either direction is intimately associated with political agitation, our endeavors in this respect will naturally fail. The increase of saloons does not increase the number of drinkers; it is the demand for alcohol that increases the number of saloons. It behooves us, therefore, to combat and endeavor to remove the morbid desire for alcohol. The principle of diminution of the number of saloons, however, should be adopted.

A more useful measure for decreasing and preventing alcoholism is propaganda in its broadest sense. The moral and intellectual influence of various antialcoholic societies is beneficial. The good they do cannot be sufficiently appreciated. Moral persuasion and conviction is most efficacious in that direction. The widest publicity concerning the effect of alcohol on physical and mental health should be considered as vital. The public should read and see the pernicious consequences of alcoholism. Lectures and clinics should be systematically organized and actual demonstrations given. The public should be invited to visit particularly institutions for the feeble-minded where they could see for themselves specimens of humanity brought into the world by parents whose alcoholic excesses have thrown on the community a useless burden. Imbeciles, idiots, and those otherwise mentally deficient, epilepties and children with all sorts of morphologic abnormalities, result from parental acquired diseases or pernicious habits of which alcoholism is one of the most conspicuous.

Propaganda against alcoholism should be particularly applied to young persons and children. The rôle of the physician is here indicated. It is he who comes in contact with families. As counselor in medical and hygienic matters he can use his great influence in various circles. Considered as a man of knowledge and experience, he will be listened to. $\mathrm{He}$ can prevent parents from giving children their first taste of drink. It is he who controls the diet and hygiene of children. It is to him that the mother addresses herself for advice as to the habits of her children.

Next to the physician is the children's teacher. But the teacher himself must be well informed as to the nature and dangers of alcoholism. $\mathrm{He}$ should have taken, before he is permitted to instruct, a special course in social hygiene in which the effects of alcoholism should occupy a prominent place. Such a course should be given in schools and colleges, and the instruction should be not only theoretical but also practical. It is necessary that every youth leaving school should possess a fundamental knowledge of the effect of alcohol on tissues, organs, the nervous system, the mentality and the offspring. From a tender age the child should be thoroughly imbued with the idea that alcohol is one of the most obnoxious and dangerous poisons. It is the knowledge of what is true, useful and indispensable in life that presents the most powerful weapon against the acquirement of pernicious habits or diseases. Imparting this knowledge constitutes real scientific prophylaxy.

'The public at large should be familiarized with the fact that one of the threatening features of alcoholism is depopulation not only quantitatively, but also qualitatively. It leads to a degeneration of both the individual and the species. It produces a slow and progressive individual deterioration and an intellectual and physical sterility of the race.

\section{THE DOSAGE OF COCAIN AND OTHER DRUGS USED FOR PRODUCING LOCAL SURGICAL ANESTHESIA}

\author{
ALBERT H. MILLER, M.D. \\ PROVIDENCE, R, I
}

Local surgical anesthetics are applied in three wavs: externally to mucous membranes, hypodermically in the region to be operated on or in the locality of the nervetrunks innervating the region of the operation, and by injection into the spinal canal. The effect aimed at in using local surgical anesthetics is local. Unfortunately, these drugs have also a general effect, which may be produced whether the drugs are administered externally to mucous membranes, hypodermically, or by injection into the spinal canal. The general effect depends on the amount of the drug which is administered, a greater effect being produced by a larger amount of the drug without regard to the strength or weakness of the solution in which it may be administered.

The toxicity of cocain is the objection to its general use as a local surgical anesthetic. The drugs which have been introduced as substitutes for cocain are less toxic, but cannot be considered as entirely safe. The extension of the idea that these drugs are safe in unlimited dose is bound to result in disaster.

The dosage of cocain is indicated exactly in the United States Pharmacopeia. The proper dose of the substitutes for cocain is generally unindicated and impossible to learn from medical literature. The dosage of these drugs is given in the literature furnished by the manufacturers in strength of solution, the inference being that any amount of a solution of that strength may be employed with safety. The dosage is calculater for the local effect without considering the general effect of the drugs.

The following series of cases illustrates the result which may come from this inexact employment of the dangerous drugs known as local surgical anesthetics. This series of cases was reported to the Providence Society of Anesthetists, Feb. 28, 1913.

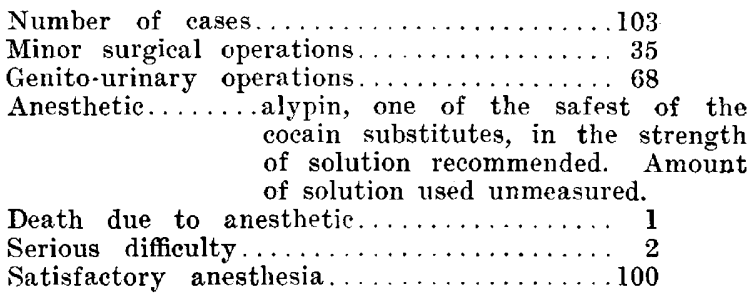


The cases of death and of serious difficulty are reported in detail. The numbers given to the cases here are not the serial numbers.

CASE 1 (June 15, 1912).-Patient, healthy man, aged 39 Operation, dilatation of stricture of urethra. Anesthetic, about 2 drams of a 10 per cent. solution of alypin, introduced through the meatus into the urethra and bladder.

About two minutes after the introduction of the anesthetic, the patient cried out and had a general muscular convulsion. During the next ten minutes there were about a half dozen such convulsions. Respiration ceased in seven or eight min. utes. At the end of ten minutes the pulse had ceased to beat. Artificial respiration and stimulation were used, but in spite of these, the patient died.

CASE 2(July 7, 1912).-Patient, healthy male. Operation, passing sounds for retention of urine. Anesthetic, unmeasured quantity of 10 per cent. solution of alypin introduced into urethra and bladder through meatus.

After about five minutes there was a general convulsion. The pulse became imperceptible. Respiration ceased. The patient was revived with difficulty after about two hours' work.

CASE 3 (Aug. 6, 1912).-Patient, apparently healthy male. Operation, dilatation of stricture of the urethra. Anesthetic, about $1 \frac{1}{2}$ drams of 10 per cent. solution of alypin introduced into urethra and bladder through meatus.

Anesthesia seemed very satisfactory. In three minutes patient became unconscious. After about five minutes there was a severe general convulsion. Respiration ceased. The pupils were widely dilated. The face was somewhat cyanotic. The pulse continued good. Treatment, artificial respiration and inhalations of oxygen. In ten minutes danger seemed to be passed. Patient recovered.

If an overdose of alypin was administered in these cases, it can be said that there is nothing in the literature of this anesthetic to indicate that the amount used was unreasonably large. The same criticism can be made of the dosage, as published, of nearly all the cocain substitutes. The revisers of the United States Pharmacopeia set the dose of cocain at $1 / 2$ grain. The dose of all the cocain substitutes should be stated as clearly as this, putting in a subordinate position any statements as to strength of solution in which the proper amount of the drug may be employed. In this way further fatalities from these very valuable products may be avoided.

279 Benefit Street.

\section{THE ELIMINATION OF GASTRIC DISTURB ANCE PRODUCED BY SODIUM CAR- BONATE, IODIDS, OIL OF SAN- DALWOOD AND SIMILAR DRUGS \\ EDGAR G. BALLENGER, M.D., AND \\ OMAR F. ELDER, M.D. ATLANTA, GA.}

For a number of years we have been working to discover some method of obviating the gastric disturbance which is produced by remedies such as potassium iodid, oil of sandalwood, sodium salicylate, sodium carbonate, etc. This we have finally and satisfactorily accomplished. Remedies, such as oil of sandalwood, creosote and oleoresins administered in soft gelatin capsules may be prevented from disturbing the stomach by the process of hardening with formaldehyd the capsules in which they are placed.
Only slight hardening is necessary, especially if the capsules are kept for some time, as additional hardening comes with age. At first we immersed the filled capsules for one minute in a dilution of 1 part 40 per cent. formaldehyd solution to from 40 to 60 parts of water. The strength should vary with the ageing allowed.

From the time that the dilution mentioned is used two weeks should be allowed to intervene before administering the capsules. A more satisfactory method of preparing the capsules is to place them in open boxes in a closed ressel in which they are subjected to the vapor of the solution of liquor formaldehydi. About 15 c.c. of the solution should be used for each cubic foot of space in the closed vessel. The solution should be placed on cotton or gauze in a saucer or tray. The time required for hardening the capsules varies with the temperature and with the time that is to be allowed before they are administered. Six hours' exposure or less is enough for capsules which are not to be administered at once, while twelve hours may be necessary in preparing capsules for immediate use. These estimates are made for ordinary soft gelatin capsules at the ordinary room temperature, from 70 to $75 \mathrm{~F}$. The capsules become hardened so that they are not digested by the gastric juice, but are digested by the intestinal secretion, if they have not been subjected too much to the vapor, in which case they may pass undigested. If dilatation of the stomach is present and the capsules or food do not pass into the intestines within the usual time, a greater degree of hardening of the capsules may be necessary. For the ordinary normal person capsules prepared as previously described will prevent gastric disturbance by carrying the medicament into the intestines before the capsules burst.

An even more satisfactory method of carrying through the stomach such remedies as sodium carbonate, potassium iodid, sodium salicylate, etc., is obtained by combining the desired remedy with mutton-suet and paraffin. When incorporated in such a mass the medicament is uniformly carried into the intestines without dissolving. as the stomach does not secrete a fat digestant. The suet is digested as it passes down the intestines and thus gradually the drug embodied in it is liberated. The paraffin is added to give additional hardness. The following combination is recommended:

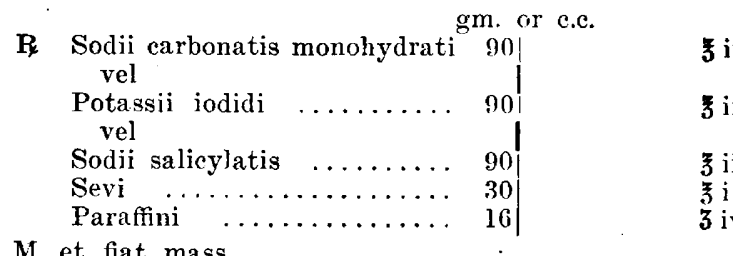

All the ingredients should be melted over water and while melted encapsulated in 00 gelatin capsules. The capsules may be filled with a fruit-spoon. The melted mixture should be of a creamy consistency, and the salt used should be previously powdered. The encapsulated product is a mass of the salt, the suet and the paraffin, is hard and remains unaffected by the gastric juice.

In the use of sodium carbonate, in the treatment of Bright's disease, as suggested by Martin H. Fischer, we have found this method of administration of decided value, as formerly it was quite difficult for the patient to take alkali in sufficient amounts to render the urine neutral or alkaline without producing gastric disturbance, with coated tongue and other symptoms.

The manner in which albuminuria and casts have disappeared under this treatment has been quite remarkable. 